\title{
Probing the Cation Distribution in Gamma-alumina Enabled by O-K Edge Artifact Suppression Using Cryo-EELS
}

Henry Ayoola ${ }^{1}$, Cheng-Han $\mathrm{Li}^{2}$, Stephen House ${ }^{1}$, Joshua Kas ${ }^{3}$, John Rehr ${ }^{3}$, Joerg Jinschek ${ }^{2}$, Wissam Saidi $^{1}$, Judith Yang ${ }^{1}$ and Cecile Bonifacio ${ }^{4}$

${ }^{1}$ University of Pittsburgh, Pittsburgh, Pennsylvania, United States, ${ }^{2}$ The Ohio State University, Columbus, Ohio, United States, ${ }^{3}$ University of Washington, Seattle, Washington, United States, ${ }^{4}$ E.A. Fischione Instruments, Inc., Export, Pennsylvania, United States

Gamma-alumina $\left(\gamma-\mathrm{Al}_{2} \mathrm{O}_{3}\right)$ is one of several transitional phases of $\mathrm{Al}_{2} \mathrm{O}_{3}$ with valuable properties that render it highly useful in a number of applications including catalysis and absorbents. Yet despite the technological importance of $\gamma-\mathrm{Al}_{2} \mathrm{O}_{3}$, its crystal structure is still not fully understood. While $\gamma-\mathrm{Al}_{2} \mathrm{O}_{3}$ was originally described as having a spinel-like structure [1], many different spinel-based and nonspinel structures have since been proposed. In our previous work, we verified that the cubic spinel-based model is indeed the most accurate for $\gamma-\mathrm{Al}_{2} \mathrm{O}_{3}$ [2]. However, the distribution of $\mathrm{Al}$ cations in the lattice remains unclear. Since cation vacancies must be added to the normal spinel structure to achieve the correct stoichiometry of $\mathrm{Al}_{2} \mathrm{O}_{3}$, the placement of the vacancies in either tetrahedral or octahedral sites determines the Al cation distribution. Prior studies have reached contradictory conclusions, with some claiming all vacancies are on octahedral sites [3], some suggesting all vacancies are on tetrahedral sites [4], and some claiming a mixed distribution [5]. Determining the cation distribution is key to accurately modeling $\gamma-$ $\mathrm{Al}_{2} \mathrm{O}_{3}$ for various theoretical simulations, including catalytic property simulations which are of great interest to the chemical engineering community.

Electron energy-loss spectroscopy (EELS) is a distinctly suitable tool for this study due to the sensitivity of the energy-loss near-edge fine structure (ELNES) to local atomic coordination. We therefore employed scanning transmission electron microscopy (STEM) and monochromated EELS combined with multiple scattering ELNES simulations to investigate the $\mathrm{Al}$ atom distribution in the spinel $\gamma-\mathrm{Al}_{2} \mathrm{O}_{3}$ structure. A major factor contributing to the uncertainty around the structure of $\gamma-\mathrm{Al}_{2} \mathrm{O}_{3}$ is the heterogeneity of commercially available $\gamma-\mathrm{Al}_{2} \mathrm{O}_{3}$. To address this, we synthesized single-crystalline $\gamma-\mathrm{Al}_{2} \mathrm{O}_{3}$ thin films through thermal oxidation of single-crystal NiAl (110). From the highly crystalline $\sim 80 \mathrm{~nm} \gamma-\mathrm{Al}_{2} \mathrm{O}_{3}$ thin films, cross-sectional TEM samples were prepared using focused ion beam (FIB) for the STEM-EELS experiments.

One of the requirements to collect accurate, high-quality STEM-EELS data is the use of sufficiently high electron beam currents. However, like many other low- $\mathrm{Z}$ oxide materials, $\gamma-\mathrm{Al}_{2} \mathrm{O}_{3}$ is highly susceptible to structural changes by the highly focused STEM electron beam. Knock-on damage as well as radiolysis effects could occur, easily observed as holes in the sample (Figure 1a) or as changes in the pre-edge fine structure in the O-K EELS edge (Figure 1b). Therefore, we first employed a carefully systematic study investigating the origin of beam effects on $\gamma-\mathrm{Al}_{2} \mathrm{O}_{3}$, in order to find optimum experimental conditions minimizing these beam effects. We found that employing cryogenic conditions - using a liquid nitrogencooled Gatan cryo-holder - was most effective in mitigating the impact of beam effects on EELS spectra; more so than reducing acceleration voltage (HT). Even under high HT conditions where knock-on damage (hole) was still present, the pre-edge peak in the O-K EELS spectrum could be completely suppressed 
under cryo conditions (Figure 1c,d). ELNES simulations were used to explain the source of the damagerelated pre-edge peak as likely due to surface $\mathrm{O}-\mathrm{O}$ bonds formed as a result of the beam-sample interaction.

Using our optimized EELS experimental conditions, Al-K, Al-L2,3, and O-K edge ELNES were acquired at cryogenic temperatures from the cross-sectional $\gamma-\mathrm{Al}_{2} \mathrm{O}_{3}$ sample to investigate the cation distribution of $\gamma-\mathrm{Al}_{2} \mathrm{O}_{3}$. $\mathrm{Al}-\mathrm{L}_{2,3}$ and $\mathrm{O}-\mathrm{K}$ edge ELNES were simulated for the differently coordinated $\mathrm{Al}$ and $\mathrm{O}$ atoms in the spinel $\gamma-\mathrm{Al}_{2} \mathrm{O}_{3}$ structure using the FEFF9 code [6]. Simulated Al-L2,3 ELNES of tetrahedral and octahedral $\mathrm{Al}$ atoms shows a clear dependence of the spectrum shape on the $\mathrm{Al}$ coordination (Figure 2a). Comparing the simulated O-K edge ELNES for the different nonequivalent $\mathrm{O}$ sites reveals that shoulder on the first peak varies in intensity as a function of the nearest-neighbor coordination around the scattering $\mathrm{O}$ atom, providing a gauge of the relative fractions of the different $\mathrm{O}$ atom environments in $\gamma-\mathrm{Al}_{2} \mathrm{O}_{3}$ (Figure 2). This is an indirect measure of the $\mathrm{Al}$ atom distribution since the $\mathrm{O}$ atom environment is dependent on the $\mathrm{Al}$ atom distribution in the lattice. Further simulations of the $\mathrm{Al}-\mathrm{L}_{2,3}$ and $\mathrm{Al}-\mathrm{K}$ edges will be presented to directly probe the $\mathrm{Al}$ cation distribution and to determine tetrahedral and octahedral Al contributions in our experimental EELS data [7].

Room temp
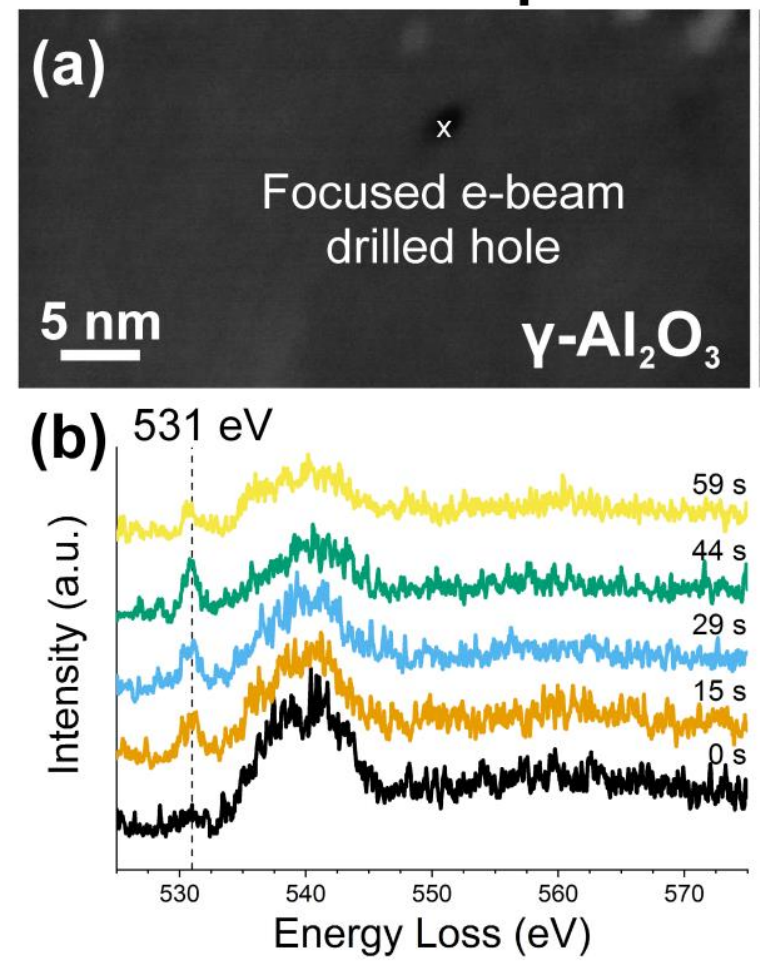

Cryo temp $\left(-186^{\circ} \mathrm{C}\right)$
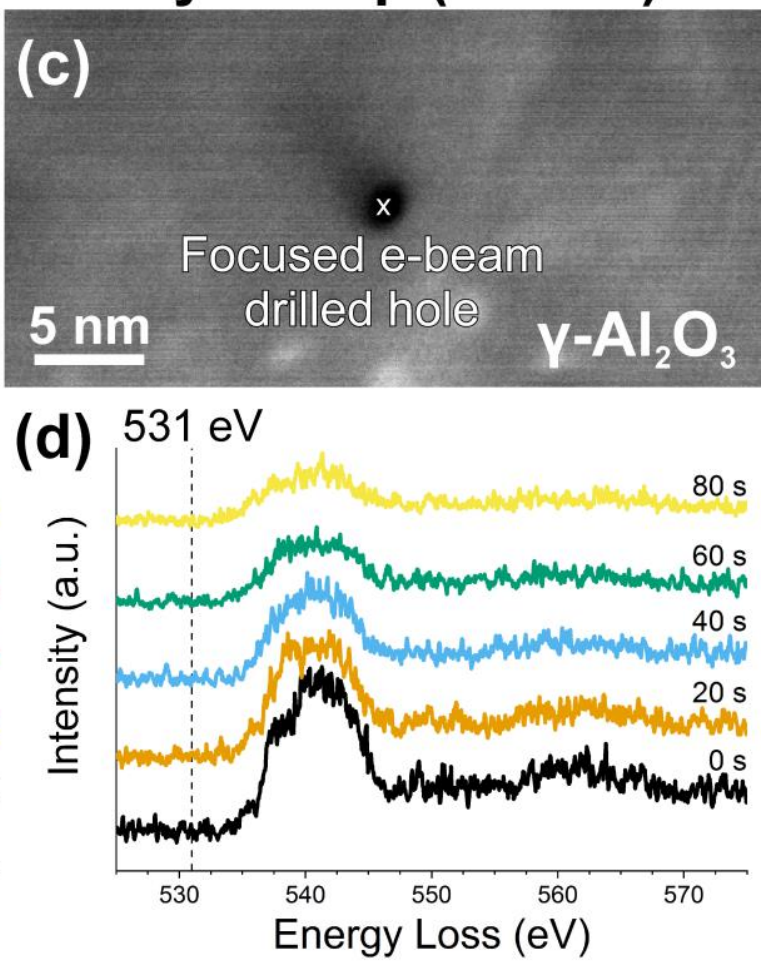

Figure 1. (a) Dark-field STEM image of $\gamma-\mathrm{Al}_{2} \mathrm{O}_{3}$ with the position where the focused electron beam was used to drill a hole marked with an x. (b) Time-resolved O-K edge EELS spectra acquired at room temperature during e-beam hole drilling. The characteristic damage-associated pre-edge peak at $531 \mathrm{eV}$ is marked with the dotted line. (c) DF-STEM image of $\gamma-\mathrm{Al}_{2} \mathrm{O}_{3}$ in the cryo experiments. (d) Time-resolved $\mathrm{O}-\mathrm{K}$ edge EELS spectra acquired at cryogenic temperature during e-beam hole drilling with no pre-edge peak observable. 

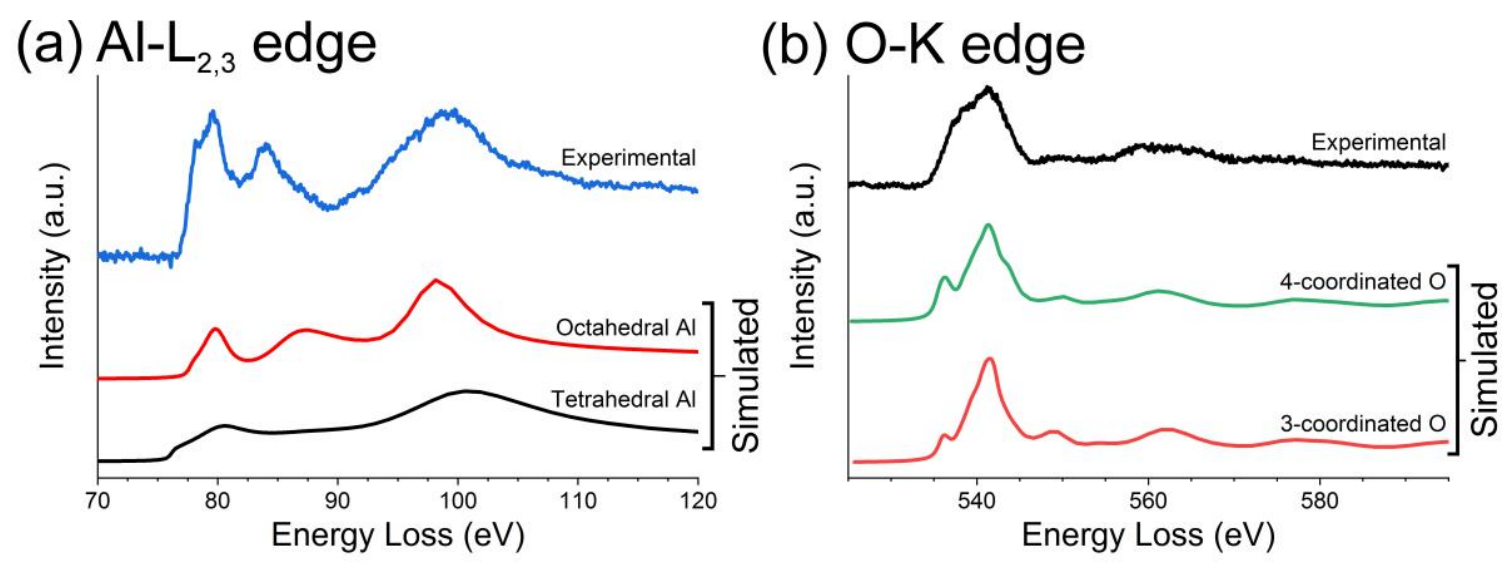

Figure 2. Comparison of experimental and simulated EELS Al-L2,3 edge (a) and O K edge (b) spectra. The Al-L2,3 edge EELS simulations were done using a single scattering shell showing the significant impact of nearest neighbor $\mathrm{O}$ atoms. The O-K edge EELS simulations were done using 4 scattering shells. Both the change in intensity of the first peak which corresponds to the peak shoulder in the experiment and the shift in energy of the second peak are correlated with the $\mathrm{O}$ atom coordination.

References

[1] E.J.W. Verwey, Zeitschrift für Kristallographie - Crystalline Materials, (1935), p. 65.

[2] H.O. Ayoola, et al., Acta Materialia, 182 (2020) p. 257-266.

[3] B. Ealet, et al., Thin Solid Films, 250 (1994) p. 92-100.

[4] V. Jayaram and C.G. Levi, Acta Metallurgica, 37 (1989) p. 569-578.

[5] L. Smrcok, V. Langer and J. Krestan, Acta Crystallographica C, 62 (2006) i83-84.

[6] K. Jorissen, J.J. Rehr and J. Verbeeck, Physical Review B, 81 (2010), 155108.

[7] The authors acknowledge support from NSF CHE-1300544, CHE-1534630 and DOE-BES (DE-FG0297ER45623). WAS acknowledges start-up funding from the Mechanical Eng. and Materials Science Dept. at U. Pittsburgh. The experimental data was acquired using instruments at the Nanoscale Fabrication and Characterization Facility (NFCF) at The University of Pittsburgh, and at the Center for Electron Microscopy and Analysis (CEMAS) at OSU. 